\title{
Recognition of topology feature of Graphene by image processing technique
}

\author{
Maofa Wang ${ }^{1}, *$, Jilin Feng ${ }^{1}$, Xiaoping Zou ${ }^{2}$, Xiaoli Li ${ }^{1}$, Pan Li ${ }^{1}$, Qingjie Liu ${ }^{1}$, Weifeng Shan ${ }^{1}$, Ying Han $^{1}$, \\ Yanping Shen ${ }^{1}$ \\ 1 Department of Information Technology \\ Institute of Disaster Prevention \\ Sanhe, 065201, China \\ 2 Research Center for Sensor Technology \\ Beijing Key Laboratory for Sensor \\ Beijing Information Science and Technology University \\ Beijing China \\ E-mail: wangmaofa2008 @126.com
}

\begin{abstract}
In order to extract characteristic values of graphene, a method based on a series of digital image processing algorithms was proposed in this paper. Using the method, we can automatically analyze topology structure and calculate amount of carbon atoms, and even calculate chiral vector indices (n, m) during in-situ TEM scanning of graphene. At last, we also write the correlative software with $\mathrm{c}^{++}$, which applying the digital image processing algorithms, and give out the processing result of several classic TEM image of graphene.
\end{abstract}

Keywords-graphene; feature values; topology structure; digital image processing

\section{INTRODUCTION}

Graphene is one of the most promising materials in nanotechnology as in [1]. The electronic and mechanical properties of graphene samples with high perfection of the atomic lattice are outstanding. Graphene is a single layer of graphite which consists of hexagonal net-like structure, and highly curved graphene derivatives such as carbon nanotubes and fullerenes with ideal lattice have been studied extensively [2]. Due to some causes, the units of the structure of TEM image of graphene are not standard hexagon, and the whole images may be compressed or distorted.

We want to find out net-like skeleton structure and calculate amount of carbon atoms in TEM images of graphene by digital image processing technique. Throughout the recognition system, there are four approaches: graying, binaryzation, thinning process, calculation of amount of carbon rings. In the paper, we give the detailed discuss and depict of the every approach above.

In the paper, we used the software to process an image of a single-layer graphene membrane (atoms appear white) coming from the article as in [2]. Although the image is a secondhand which has degraded, and is not clear as the original image, we also can get an ideal result.

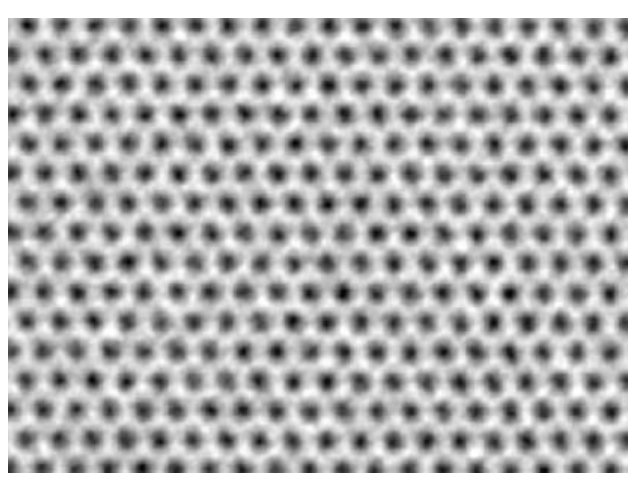

Figure 1. A typical TEM image of graphene.

\section{Key AlgORITHMS ANALYSIS AND DisCUSSION}

\section{A. Image Graying}

In the first place, we need to transform the TEM image (Figure.1) from color pattern to gray-scale, because of that an available gray scale image is essential preparation for binarization processing late as in [3-4]. There is only brightness information in grey scale image, but not color information. In this approach, there are 8 bits per pixel in one new image corresponding to 24 bits per pixel in the color pattern image where per 8 bits denotes the weight of one kind of color. By Eq. (1), we can obtain the transformation relation between these two pattern images.

$$
G=0.30 r+0.59 g+0.11 b
$$

Here $G$ is the brightness value of one pixel in the new gray image, whose corresponding color brightness (red, green, blue) values in color image are $r, g, b$, separately. Figure. 2 is the result of converting Figure.1 from color pattern into gray-scale. 


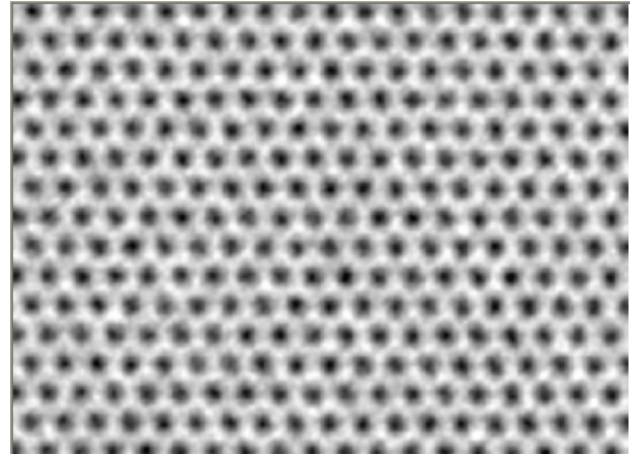

Figure 2. The result of converting Figure.1 from color pattern into gray pattern. Although Figure.1 and Figureure. 2 look alike but in image pattern they are very far apart from each other.

\section{B. Image Binarizing}

Binarization is a digital image processing algorithm by which one image can be divided into two parts: object part and background part as in [3-4]. Binarization processing is a very important approach to recognition of atomic structure of graphene. To partition one image by magnitude of grayscale is the most popular binarization method. If one pixel value of original image is $f(x, y)$, we can choose one grayscale value $t$ as partition threshold by one kind of certain algorithm, then determine one pixel point as object point when its original pixel value is not less than the value of $t$ and give the point a new pixel value of 1 , or else determine one pixel point as background point whose original pixel value is less than of $t$, and give it a new pixel value of 0 . By Eq. (2), we can obtain the conversion relation between new and original pixel value.

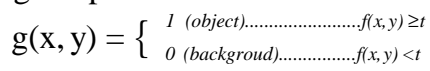

The key-problem is how to obtain partition threshold value of an image. In this paper, we use maximum entropy method to calculate partition threshold.

In information theory, we define the entropy as the uncertainty of Probability distribution. For a system which has $n$ states: $\varepsilon_{0}, \varepsilon_{1}, \ldots, \varepsilon_{n-1}$, and the corresponding probabilities is: $p_{0}, p_{1}, \ldots, p_{n-1}$, whose system entropy is defined as:

$$
H\left(p_{0}, p_{1}, \ldots, p_{n-1}\right)=-\sum_{0}^{n-1} p_{i} \ln p_{i}
$$

The greater the entropy is, the less the information held in a system is. On the contrary, the smaller the entropy is, the greater the information is. In one image, one area with greater entropy will have relative even gray values; and the discreteness of gray values will be bigger in one area with smaller entropy. In one graphene image, object areas are blended with backgroud areas, and the gray values in different areas are relative even. So we can extract and segment the object points from the background points based on maximum entropy in one graphene whose gray values are relative even.

Based on the definition of the information entropy, we can get entropy image define as Eq. (4).

$$
H_{T}=-\sum_{i=0}^{l-1} p_{i} \ln p_{i}
$$

Here $p_{0}, p_{1}, p_{2}, \ldots, p_{l-1}$ are gray probability distributions in one image.

Let $t$ is one gray value which divided all the gray value into two classes: $\{0,1, \ldots, \mathrm{t}\}$ and $\{t+1, t+2, \ldots, l-1\}$. And the occurrence probability of one gray value in corresponding class is:

$$
\begin{gathered}
\text { A: } \frac{p_{0}}{P_{t}}, \frac{p_{1}}{P_{t}}, \ldots, \frac{p_{t}}{P_{t}} \\
\text { B: } \frac{p_{t+1}}{1-P_{t}}, \frac{p_{t+2}}{1-P_{t}}, \ldots, \frac{p_{t+2}}{1-P_{t}} \\
P_{t}=\sum_{i=0}^{t} p_{i}
\end{gathered}
$$

Let $H_{A}(t)$ is of the entropy of class $\mathrm{A}$, and $H_{B}(t)$ is of the entropy of class $\mathrm{B}$, then $H_{A}(t)$ and $H_{B}(t)$ are defined separately as the following:

$$
\begin{aligned}
H_{A}(t) & =-\sum_{i=0}^{t} \frac{p_{i}}{P_{t}} \ln \frac{p_{i}}{P_{t}}=\ln P_{t}+\frac{H_{t}}{P_{t}} \\
H_{B}(t) & =-\sum_{i=t+1}^{l-1} \frac{p_{i}}{1-P_{t}} \ln \frac{p_{i}}{1-P_{t}}=\ln (1-P)_{t}+\frac{H_{T}-H_{t}}{P_{t}}
\end{aligned}
$$

Let $H(t)$ is the total entropy of one image which is the sum of $H_{A}(t)$ and $H_{B}(t)$ :

$$
H(t)=\ln P_{t}\left(1-P_{t}\right)+\frac{H_{t}}{P_{t}}+\frac{H_{T}-H_{t}}{1-P_{t}}
$$

When A highest $H(t)$ values was obtained, $t$ is used as the optimal threshold: thre which divide one image into object part and background part.

$$
\text { thre }=\operatorname{ArgMax}\{\mathrm{H}(\mathrm{t})\} \quad(0<t<l-1)
$$

Figure. 3 is the last binarization processing result.

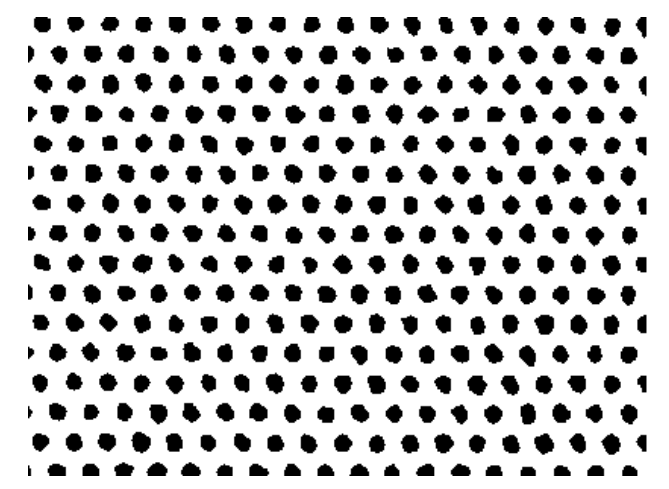

Figure 3. The result of the binarization based on maximum entropy method. 


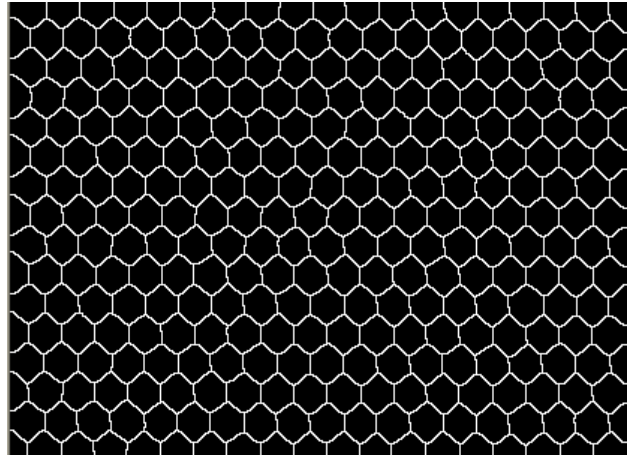

Figure 4. The result of thinning of the Figure. 3.

\section{Mathematical morphology as a thinning process}

Here, some morphological operations as in [3-4] are given. Let $S$ be the set of an image and $B$ be the set of structure elements (or templates), which are subsets of the universal space $E$. Then,

- Minkowski addition

$S \oplus B=\{z \in E: z=s+b$, for some $s \in S$ and $b \in B\}(12)$

- Minkowski subtraction

$S \Theta B=\{z \in E: z+b \in S$, for some $s \in S$ and $b \in B\}$

- Hit/miss transform

Let $B$ be composed of two subsets $B_{1}$ and $B_{2}$ where $B_{1}$ is the foreground and $B_{2}$ is the background, then,

$$
S \otimes B=\left\{s: B_{1} \subset S ; B_{2} \subset S^{c}\right\}=\left(S \Theta B_{1}\right) \cap\left(S \Theta B_{2}\right)
$$

Here $S^{c}$ is the complement of $S$. Hence, the hit/miss operation can detect the exact location of the pattern $B$ occurring in the image $S$.

- $\quad$ Thinning of $S$ by $B$

$$
S \circ B=S /(S \otimes B)
$$

Here, the operator / is the set difference operation.

Thus, thinning can be considered as a search-delete process using a particular pattern $\mathrm{B}$, in the image $\mathrm{S}$.

A modified thinning algorithm based on the morphological hit/ miss transform was described in [8].

The result of thinning of the Figure. 4 is as shown in Figure. 5. From the Figure. 4, we can clearly see the hexagonal topological structure of the graphene membrane.

\section{Recognition of amount of carbon atoms}

In order to recognize of amount of carbon atoms, there are six approaches: graying, binaryzation, Distance transform as in [5-6], Seed points searching, calculating the sum of atoms. It is must be pointed out that graying and binaryzation have been described by the above section $B$ and $C$, and the thinning process is not need in Recognition of amount of carbon atoms.

- $\quad$ Distance transform

A binary image can be represented by its object set $F$ and background set $F^{c}$. The distance transform of the object set $F$ is also a gray image with values at each pixel

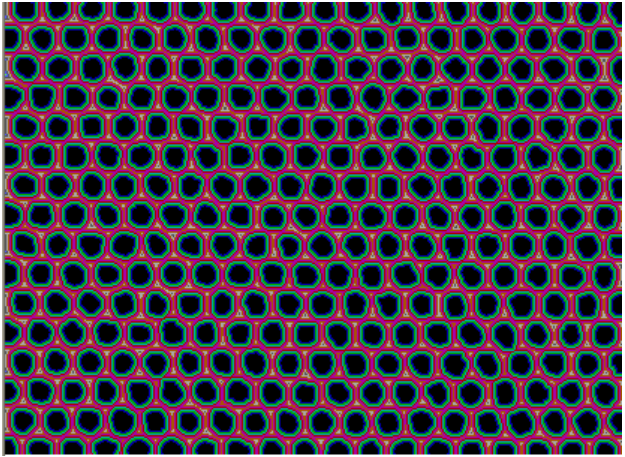

Figure 5. The result of distance transform.

point representing its distance to the nearest pixel point of set $F^{c}$. It is defined by Eq. 3 .

$$
D(p)=\min \left(\operatorname{dist}(p, q), p \in F, q \in F^{c}\right)
$$

Here $p$ is one pixel point which is in set $F$, and $q$ is another pixel point which is in set $F^{c}$. dist is the distance function which is used to measure the distance of two different points.

The detail distance transform method is described in [4]

\section{- Seed points searching}

There are some core areas which have local maximum distance values in the distance transform image as in Figure. 5. The sum of the core areas represents the sum of carbon rings in Figure. 1. All the pixel points within one core areas have the same gray value which is greater than the gray value of all the adjacent pixel points outside the core region.

The key question is to how to find out core regions. Firstly, we will scan each one line from top to bottom in distance transform image (as in Figure. 5) to obtain the all the local line segments which have maximum pixel value in per line. The collection of the local line segments having the same maximum distance value in the adjacent lines forms the above referred core regions.

In each core region, there is commonly one seed point which is defined as the left point of the bottom line [4], so the sum of the seed pints represents the amount of the carbon rings in the graphite TEM image. But because of that the some core regions exist with very complex shape, or there are holes in some core regions, or some core regions are adhesive with other core regions, so there are some core regions having more than one seed point. In other words, we can not directly give out the sum of the hexagonal carbon rings in the TEM image( as in Figure. 1 ) by calculating the amount of the seed points in the distance transform image (Figure. 5), and must eliminate any redundant seed points to make sure that there is only one seed point in one core region. This can be completed by checking the coordinate distance of between every potential two seed points in one core region, to see the coordinate distance between them is greater than the maximum distance value of the two seed points. If the result is not, only leaving the seed point which has larger distance value. 


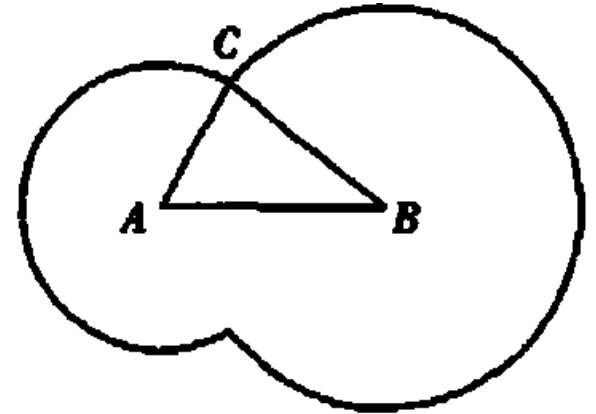

Figure 6. The sketch map of one region where there are two seed points.

Figure. 6 is the sketch map of one core region where there are two seed points: point $A$ and point $B$, and Point $C$ is boundary point of the core region. $A C$ and $B C$ are the distance values of the two seed point, respectively. $A B$ is the coordinate distance between the two seed point. The detailed judgement algorithm of redundant seed point is decribed as the follow:

$$
\begin{aligned}
& Q=\max (A C, B C)+1 \\
& \text { if } A B<Q \text { the } T=\text { true } \\
& \text { else } T=\text { false }
\end{aligned}
$$

Here, if $T=$ true, $A$ and $B$ are in the same core region, and only leave the seed point which has larger distance value. Not that, do nothing.

Figure. 7 shows the result image of searcing seed points, we can clearly see that each one core region has one seed point. Here, the sum of seed points has the value of 300 which is automatically given out by the software with C++ codes as in [7], and is very closed to the expected sum of the carbon rings in Figure. 1.

\subsection{Calculating the sum of the carbon atoms}

By analyzing the net-like hexagonal topological structure of graphene, it is easily found that one carbon ring actually contains two carbon atoms. And the relation between the amount of the carbon atoms in graphene image (as in Figure.1) and the sum of the seed points(or core regions ) in Figure. 7 is defined by Eq. (17).

$$
m=2 * n
$$

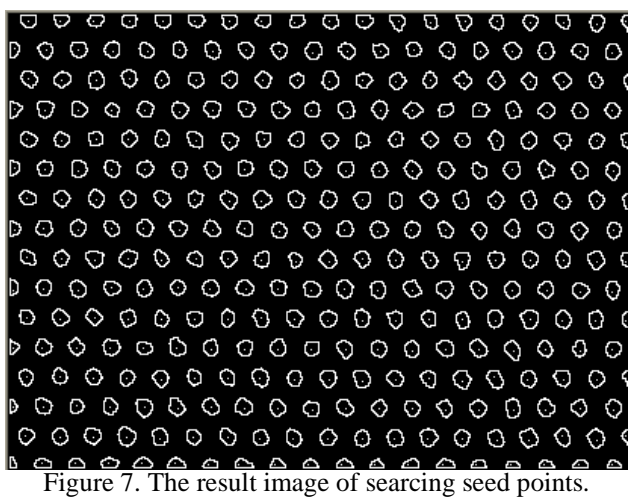

Here $m$ is the the amount of the carbon atoms, $n$ is the sum of the seed points. For the TEM image (as in Figure.1), the value of $n$ is 300, so we calculte the value of $m$ is about 600 which denotes the amount of the carbon atoms in Figure. 1.

\section{CONCLUSION AND PROSPECT}

The present study have gotten topological structure calculated and calculate out amount of carbon atoms in graphene images which is hopeful to analyze surface structure of graphene during In-situ STM scanning of the analyzed object later.

Our next job is to improve algorithm and write the soft which we want to use to recognize defect structure of graphene automatically. And the correlative study is going on now in our team.

\section{ACKNOWLEDGMENT}

Two of Authors (Maofa Wang, Jing Yuan) thank the financial support from Special Fund of Fundamental Scientific Research Business Expense for Higher School of Central Government (Projects for young teachers) (Recognition of defect structure of graphene based on HRTEM and STM images: ZY20110212)

\section{REFERENCES}

[1] Florian Banhart, Jani Kotakoski, and Arkady V. Krasheninnikov,"Structural Defects in Graphene," ascNano, vol. 5(1), pp. 26-41, 2011.

[2] Jannik C. Meyer, C. Kisielowski, R. Erni, Marta D. Rossell, M. F. Crommie, and A. Zettl, "Direct Imaging of Lattice Atoms and Topological Defects in Graphene Membranes,” NANO LETTERS. vol. 8(11) pp. 3582-3586, 2008

[3] Wang, M.F, Zou,X.P, "Recognition of atomically-resolved STM images of graphite, " Journal of Computational and Theoretical NanoScience. vol. 7 pp. 404-407, 2010.

[4] M. F. Wang, J. L. Feng, J. Y, F. P. Gao, X. P. Zou, Y. H. Yang "Recognition of amount of carbon atoms of graphite STM," Journal of Computational and Theoretical NanoScience. vol. 8, pp. 22042208, 2010.

[5] M. A. Butt and P. Maragos, "Optimum design of chamfer distance transforms," IEEE Transactions on Image Processing. vol.7, pp. 1477-1484,1998.

[6] “Distance transform”, Wikipedia internet web, the free encyclopedia. last modified on 21 December 2010.

[7] Z. Lu, “Image processing programming with c/c++," Tsinghua university press, Beijing, 2006. 